\title{
Téoros
}

Revue de recherche en tourisme

\section{L'image du Québec vu par ses brochures}

\section{Caroline Méthot}

Volume 10, numéro 2, juillet 1991

Accueil et tourisme

URI : https://id.erudit.org/iderudit/1078960ar

DOI : https://doi.org/10.7202/1078960ar

Aller au sommaire du numéro

Éditeur(s)

Université du Québec à Montréal

ISSN

0712-8657 (imprimé)

1923-2705 (numérique)

Découvrir la revue

Citer cet article

Méthot, C. (1991). L’image du Québec vu par ses brochures. Téoros, 10(2), 39-41. https://doi.org/10.7202/1078960ar d'utilisation que vous pouvez consulter en ligne.

https://apropos.erudit.org/fr/usagers/politique-dutilisation/ 


\section{L'image du Québec vu par ses brochures}

Dans le cadre d'une réflexion sur l'accueil des visiteurs, l'examen de brochures promotionnelles et publicitaires offerte aux touristes semble selon nous des plus pertinentes.

En effet, lorsque le voyageur consulte des brochures touristiques, il développe, avant son départ, une certaine image du pays ou de la région qu'il prévoit visiter. Cette image touristique est définie par Lanquar ${ }^{(1)}$ (1985) de la façon suivante:

Une image existe s'il y a un minimum de connaissances sur un pays. Addition d'un certain nombre d'images fractionnées, et propres à certains publics, l'image touristique n'est toutefois que l'un des aspects de l'image générale d'un pays. Aussi ne peut-elle jamais être très différente de la réalité de ce pays. (...) Avant le voyage, le touriste a, du pays, une image imaginée. Cette image intellectuelle, positive est la résultante d'une lecture, de rếférences d'amis. Un minimum de connaissances conditionne donc l'existence de cette image indirecte. (...) Après le voyage s'inscrit dans la mémoire du touriste l'image vếcue. C'est avant tout une image physique, directe, basce sur des $c 16$ ments sensoriels du type: la rue est animée la nuit (ouile), cette vue est imprenable (vue), etc. L'image imaginée doitêtre proche de l'image vécue. C'est aux responsables du tourisme de réduire l'écart entre la réalité et les aspirations des clients potentiels.

Pour montrerl'importance que revêt l' image imaginée, Lanquar ${ }^{(2)}$ citeégalement l'extrait suivant d'une étude menée par D.J. Bourstin (1971):

L'intérêt que nous portons à un pays étranger n'est bien souvent que la curiosité de vérifier si notre impression est conforme aux images offertes par les journaux, les films, la télévision... Nous n'allons pas confronter l'image à la réalité, mais juger la réalité d'après son image.

Madame Caroline Méthotestétudiante à la maitrise en anthropologie a I'Universite de Montreal.
Dans ce mêtme ordre d'idée, le questionnement de Roger Nadeau (3) (1982) sur les symboles est tout aussi pertinent:

Pourquoi pour l'Européen moyen, l'Amérique c'est les Indiens, New York et les chutes Niagara? Pourquoi le symbole ou l'image qui identifie le Canada dans le monde entier, est celui de la Police Montée?

Il semble donc que ces symboles et ces images soient construits de toute pièce. Cet article résume les grands points d'une etude $^{(4)}$ visant à comprendre comment le ministère du Tourisme du Québec, via quelques-unes de ses brochures touristiques destinées au public québécois et ếtranger (ontarien, américain, européen, etc.) construit une certaine image touristique du Québec.

\section{La problématique de recherche}

Le point de départ de notre réflexion fut de considérer que l'image touristique (imaginée) peut être en bonne partie influencée par la lecture de brochures publicitaires conçues par le ministère du Tourisme pour vendre le Québec comme destination touristique. Force fut également de considérer que toute publicité, apparemment centréce sur un produit (ici le Québec comme destination touristique), développera en plus, comme en prime, une vision de l'homme et du monde. A ce sujet, les propos de L. Bardin $^{(5)}$ (1975) sont explicites:

\section{L'image et le texte publicitaire décrivent leur propre vision du monde et renvoie à une réalité tron= quée. Par le biais de l'objet promu, autour -ou, en quelque sorte, en sur- impression $=$, le petit monde de la publicité attribue un sens aux choses et aux actes, renvoie à une philoso- phie de la vie, diffuse tout un sys- tème de valeurs.}

Il ne va pas alors sans penser que le contenu des brochures publicitaires sur le Québec fait appel à des jugements, des valeurs ou mềme à une certaine idéologie de ceux et celles qui les conçoivent (dans notre cas, le gouvernement du Québec). Dans ce sens, les réflexions de Lerivray ${ }^{(6)}(1975)$ au sujet de l'idéologie contenue dans les guides Bleus et Verts de Michelin sont des plus intéressantes:
Il faut, en effet, faire des choix, opérer une sélection, orienter le voyage, proposer des lieux de séjour (...). Ces idées, ces jugements, ces choix, ces valeurs représentent un ensemble plus ou moins cohérent, plus ou moins organisé, un systeme, une doctrine, une «idéologiew. (...) Les guides Bleus et les guides Verts opèrent ces choix à partir de critùres qui leur sont propres, à partir de normes fixées par eux à l'avance, à partir de ce qu'ils considèrent, dans leur doctrine, comme devant être vu.

Notre but n'a pas été de mener une étude détaillée du contenu idéologique, mais plutôt de rếpondre à une question générale que nous avons posée comme suit: Quelle image le ministère du Tourisme véhicule-til du Québec et des Québécois dans ses brochures touristiques?

Bien sûr, pour répondre à une question aussi vaste, nous avons d'abord apporté des réponses à plusieurs questions spécifiques, dont celles-ci que nous avons retenu pour les fins de cet article.

1- Comment le Québec est-il vendu en tant que destination touristique? Estil vendu comme un ensemble formé de régions touristiques ou comme un ensemble de produits touristiques?

2- Le ministère du Tourisme a-t-il un positionnement précis pour vendre le Québec à un groupe de touristes en particulier? Par exemple, vend-il le Québec aux Américains de la même façon qu'il le fait aux Français?

3- Utilise-t-on dans les brochures publicitaires un langage décoratif pour décrire les intérểts touristiques.

4- Comment présente-t-on la sociếté et la culture québécoise dans la brochure Québec. L'autre Amérique? Quel portrait fait-on de la population québécoise?

Évidemment, pour toutes ces questions, nous avons émis des hypothèses qui ont été par la suite vérifiées. Nous avons également, dans le but d'effectuer une meilleure opérationnalisation, définit plusieurs con- 
cepts. Ces étapes sont toutefois trop nombreuses pour en faire part dans cet article.

\section{Le corpus}

Le ministere du Tourisme publie de nombreux dépliants et brochures touristiques sur le Québec. Il était impossible - et non nécessaire - de les étudier tous pour répondre à notre question générale. Nous avons choisi comme échantillon pour notre étude les brochures Vacances d'été (Destination Québec), Vacances $d$ hiver (Destination Québec) et Québec. L'autre Amérique.

Ces trois brochures ont la particularité de donner une vue d'ensemble de la destination tout en ciblant les marchés américain, européen et québécois. De plus, nous croyons qu'elles sont assez représentatives de l'image que le ministère du Tourisme souhaite véhiculer du Québec.

\section{La méthode}

Nous ne prétendons pas ici avoir mené une analyse de contenu telle que définie par Berelson etcité dans Bardin (1977): «Une technique de recherche pour la description objective, systématique et quantitative du contenu manifeste des communications, ayant pour but de les interpréter», parceque pour être valide, selon Bardin (1977, p. 36), une telle analyse doit minimalement être d'une part exhaustive (notre but n'était pas d'épuiser la totalité du contenu) et objective (nous avons procédé de façon intuitive).

N'étant pas exhaustive et objective, notre analyse reste personnelle. Cette étude ayant été avant tout de type exploratoire, nous n'avons pas la prétention de considérer que nos observations doivent être partagées par les lecteurs. Par conséquent, les conclusions qui seront mentionnées ici ne représentent qu'une approche de réponse à la question complexe poses: Quelle image le ministêre du Tourisme vêhicule-t-il du Québec et des Québécois dans ses brochures touristiques?

\section{Derrière les stratégies de vente}

Selon les sources consultées ${ }^{(5)}$, pour vendre le Québec comme destination touristique, le ministere du Tourisme a choisi de conjuguer deux approches: 1'approche dite horizontale et l'approche dite verticale. La première consiste à assurer le positionnement global du Québec, c'est-à-dire promonvoir le Québec dans son ensemble. C'est à partir de 1988 que $1^{\prime}$ approche verticale par produit est venue s'y greffer. Celleci vise non pas à offrir aux consommateurs (touristes) des destinations (desrégions par exemple), mais plutôt des produits précis. Ainsi, selon la furme Samson Bélair (1988), dans les campagnes publicitaires, «les produits offerts devront être dominants, mais à la fois enrobés dans l'image de la destination globale, l'image faisant partie de l'achats,

En examinant cette stratégie de vente et en procédant également à une analyse des illustrations des brochures, on peut dire que le ministère du Tourisme donne l'image que le Québec est un endroit touristique où l'on peut vivre l'expérience urbaine des GRANDES VILLES, visiter quelques régions en choisissant le CIRCUIT de son goût, se livrer à une vaste gamme d'activités grâce à un SÉJOUR DE VILLÉGIATURE, se rassembler entre membres à l'occasion de CONGRES OURÉUNIONS, pratiquer le sport d'hiver par excellence: le SKI ALPIN, tenter l'expérience de la CHASSE ET DE LA PECHE, partir à la découverte des nombreux sentiers aménagés pour la MOTONEIGE ouencore profiter des grands espaces québécois en partant à 1'AVENTURE.

\section{À chacun son image}

L'analyse des différents positionnements suivant les marchés visés nous montre que le Québec c"est aussi la découverte de l'exotisme, du spectaculaire, du plaisir et de la diversité. On se trouve donc en présence d'une destination qui a l'avantage d'avoir différents visages pour ainsi plaire à différentes clientèles.

Par exemple, pour promouvoir la destination Québec en Ontario et aux États-Unis, le ministère du Tourisme a choisi de mettre en évidence la spécificité culturelle et patrimoniale du Québec. Ces concepts, très vaguement définis, semblent regrouper des aspects tels que la langue, la gastronomie, le cachet francophone, etc. On mentionne à ce sujet dans le rapport Samson Bélair("(9) que c'est la ville de Québec avec sa localisation, son concept aintra-muross et son avenue Grande Alléequi symbolise le mieux cette spécificité.

Il nous est alors apparu que le mot clé pour résumer ce positionnement soit celui de différence puisque selon la firme Samson Búlair, ala spécifícité culturelle et patrimoniale du Québec est unique en Amérique du Nordetconstitue l' élément différentiel qu'il faut afficher clairement et constamment». Il est intéressant aussi de noter que la firme a introduit ce positionnement en le situant par rapport au phénomène du tourisme. «En tourisme, la différence s'appelle souvent exotisme. Sur le continent américain, le Québec peut être perçu comme exotique au même titre que le Mexique, en mettant en évidence sa différence de culures.

Pour promouvoir le Québec sur les marches à l'outre-mer (France, Belgique, Suisse, Japon, Allemagne et Grande-Bretagne), le ministère du Tourisme a choisi cette fois-ci de metre en évidence laccessibilité des grands espaceset lefleuve St-Laurent. Dans ce positionnement, c'est l'aspect spectaculaire qui a retenu l'attention. On dira du fleuve St-Laurent qu'il est majestueuxeton insistera sur le fait qu'il s'agisse d'un des plus grands au monde avec le Danube et I'Amazone.

Pour ce qui concerne la clientèle québécoise, le ministère du Tourisme insiste sur la diversité et le plaisir de la destination. Ce positionnement a été introduit comme suit: a Sur des thèmes tels que Quatre Saisons/ Quatre plaisirs, le Québec doit se positionner sans complexes auprès des Québécois comme un pays doté de ressources au niveau touristique très atrayantes*.

On a donc ici trois marchés cibles avec trois positionnements fort différents pour décrire la même destination... A chacun son image!

\section{C'est une langue belle}

Pour établir s'il y avait utilisation d'un langage décoratif dans les brochures à notre étude, nous avons délimité un échantillon de 6 pages par brochure (environ $10 \%$ du contenu) pour lesquelles nous avons procédé à un calcul de fréquence des qualificatifs dits décoratifs. Nous entendons ici les qualificatifs (adjectifs et adverbes surtout) qui expriment un jugement de valeur, dans la plupart des cas positifs, sur les choses à voir sans toutefois leur apporter une précision réelle (par exemple dire d'un site qu'il est beau ou agréable, d'une route qu'elle est jolie). Pour mener cette étude, nous avons emprunté la méthode qu'a utilisé $B$. Leriveray (1975) ${ }^{(10)}$ pour analyser le langage décoratif des guides Bleus et Verts de Michelin.

Ainsi, nous avons relevé à $\mathrm{l}^{\text {tintérieur des }}$ six pages sélectionnées de façon aléatoire de la brochure Vacances d'été, 108 qualificatifs, soit une moyenne de 18 par page. La brochure Vacances d'hiver contenait pour sa part 115 qualificatifs (19 par page). Les qualificatifs les plus utilisés sont, par ordre d'importance: grand, vaste, bon, immense, vieux, beau, facile et abondant.

Avec de tels résultats, on peut affirmer qu'une bonne partie des intếrêts soulignés (par exemple le fleuve, les villes, les paysages, les églises, etc.) sont accompagnés d'une épithète destinée à les valoriser. On dit par exemple des eaux majestueuses, un decor splendide, des panoramas saisissants. A cela, il faut ajouter aussi les noms décoratifs comme paradis des skieurs, paysages de cartes postales, eden du plein air, lesexpressions verbales décoratives du type ne peuvent résister, faire l'envie de, n'ont que l' embarras du choix; les intérêts touristiques auxquels on a conjugué 2 qualificatifs à la fois: vastes espaces sauvages. ville animée et active; et enfin l'abondance des superlatifs dans les phrases comme: un 
des parcours les plus populaires du pays, le meilleur réseau de sentiers aménagés du monde, etc.

Il ne va pas sans dire que ce langage décoratif laisse apparaître une image fort séduisante de la destination. Le Québec devient un endroit de rêve qui frappe par la grandeur et la beauté de ses attractions. On invite ainsi le visiteur à découvrir un décor ruralenchanteur, une nature généreuse, un territoire pittoresque et séduisant tout en profitant d'une hospitalité sans pareille.

\section{Représentation de la société et de la culture québécoises}

Pour répondre à notre dernière question, nous avons procéde à une analyse thematique qualitative. C $\mathrm{C}^{\dagger}$ est-ầ-dire que nous avons, pour chaque thème contenu dans notre hypothèse (par exemple la représentation des autochtones), constitué un montage des photographies utilisees dans la brochure Québec. L'autre Amérique s"y rapportant. Malheureusement, cette partie *visuelles de l'étude ne peut être contenue dans cet article.

Nous avons choisi d'utiliser cette brochure d'abord pour ses images et ses textes peu nombreux qui nous permettaient d'analyser son contenu presque en entier, parce qu' elle donne de façon concise une vue d'ensemble du Québec et parce qu'elle est destinée uniquement à un marché outre-mer. Elle est d'ailleurs publiée en français, anglais, allemandet japonaisetest distribuée par les bureaux des délégations générales du Québec à l'étranger (Bruxelles, Tokyo, Dusseldorf, etc.). Nous avons utilisê l'édition de 1988.

\section{Le Québec à l'américaine}

De toute évidence, dans la brochure $Q u \ell-$ bec. L'autre Amérique, on a voulu montré leQuébec comme étant d'abord une région de l'Amérique du Nord. Il faut noter à ce sujet que dans presque aucun cas on a fait référence au Canada dans son ensemble ou aux autres provinces. Le positionnement se fait toujours par rapport à l'Amérique: berceau de l'Amérique française, la vieille Europe bat toujours au coeur de la jeune Amérique, si on y vit à l'américaine, on y conserve un caractère bien distinct, etc. En insistant sur le caractère nord-américain du Québec, on a ainsi présentế la sociétét québécoise comme étant avant tout moderne, neuve.

\section{La différence québécoise}

Ce qui semble caractériser cette société québécoise de cette Amérique serait sa différence culturelle. On évoque à ce titre l'importante spécificité cuiturelle, 1e caractère bien distinct ou encore la différence toutcourt: "AuQuébec, l'Amérique affirme sa différence quand, au détour đ'un chemin, elle vous dit bonjour! * Mais cette différence culturelle, comment est-elle représentée? Nous avons observé de façon assez évidente quatre «facettes $»$ de ce qui pourrait illustrer le concept de différence culturelle.

Une première facette de cette différence québécoise est sans contredit la pratique linguistique: Car le Québec, c'est aussi une langue, le français, parlé par cinq millions de gens dans un contexte de 250 millions d'anglophones; le Québec, c'est le bonjour de l'Amérique. C'est également une gastronomie différente. On y mange une fine cuisine en plus d'une cuisine typiquement québécoise: fine cuisine française, mais aussi une nouvelle cuisine québécoise; try $a$ tourtière aux trois viandes, bison tongue, glace au pralind'érable or blueberrypie. On insiste également sur l'histoire qui nous rappelle surtout son passé colonialiste français: ses importantes fortifications, ses rues abruptes et ses ruelles, ses places et jardins publics, ses escaliers pittoresques témoignent de son riche passé. Et enfin, on souligne une architecture qui témoigne d'une évidente influence européenne: $D e ́$ couvrir l'architecture québécoise, c'est entrevoir les origines variées de ses habitants; demeures seigneuriales qui portent gravée dans leurs pierres la mémoire de cette Amérique qui fut jadis française, etc. Voici done comment dans cette brochure le concept de adifférence culturelle» est représenté.

\section{Des Québécois pure laine}

Le Québec est aussi présenté comme une sociêté qui reconnaît, dans les faits, la diversité ethnique de sa population: Quebec is a society open to the world and to all cultures. French is its official language, but a multitude of tongues are spoken there, testifying to the cultural diversity of its native people and immigrants from more than 80 countries. Nous disons udans les faits» puisque cette diversité ethnique est reconnue (suivant les textes), mais une analyse des illustrations nous permet de constater que cette même diversité $n^{4}$ est pas montree. En effet, la grande majorité des personnes apparaissant sur les photographies présentent des caractéristiques physionomiques que l'on serait porté à attribuer à des aQuébécois pure laines. De toute évidence, les populations antillaise, africaine, asiatique et amérindienne ne sont pas représentées. On note une seule exception: on a pris soin de placer une photographie d'un jeune garçon Inuit à côté d'un texte faisant réferrence aux chasseurs Inuits et aux trappeurs Amérindiens. Ceci nous a porté à réfléchir sur la représentation des autochtones dans les brochures et nous sommes venus à la conclusion que d'abord une seule photo ne suffisait pas pour parler de représentation et que, malheureusement, en examinant les brochures Vacances $d^{\prime \prime h i v e r ~ e t ~ V a c a n c e s ~ d ' e ́ t e, ~ o n ~ p e u t ~ p e r c e-~}$ voir que les autochtones semblent être présentés comme des intérêts touristiques. Ainsi, on peut voir le portrait d'une jeune femme Inuit portant son bébé dans son dos, juxtaposé à une photographie d"un barrage à la Baie James... avec un peu d'imagination on comprend qu'il s'agit là de deux attractions pour le prix d'une!

Enfin, notons que les Québecois présentés dans la brochure Québec. L'autre Amérique sont bien plus que des gens accueillants et chateureux. On dit d'eux qu'ils ont de l'originalité, qu'ils sont andacieux, talentweux ${ }_{\text {, }}$ qu'ils ont le sens de l'organisation, et bien plus encore.

\section{Par-delà les brochures}

Nous voilà ici avec une image du Québec et des Québécois telle que façonnée par le ministère đu Tourisme et présentée dans trois de ses brochures. Il reste à vérifier maintenant si cette image correspond à celle que les touristes se font du Québec. II serait tout aussi intéressant de confronter cette image imaginé d' l'mage vécue telle que définie par Lanquar. Des touristes pourraient, par exemple, être interrogés à leur arrivéé ainsi qu'à leur départ. Ceci permettrait de voir, entre autres, quel roble joue l'image imaginée au cours du voyage.

\section{Par-delà les images}

Dans ce sens, les personnes responsables de l'accueil touristique doivent prendre conscience que le voyageur arrive au pays avec une certaine image (qu'il a construite à partir de diverses sources d'information) et qu'il importe que le touriste puisse aller au-delà des images parfois fausses.

\section{Notes explicatives}

(1) LANOUAR, Fobert, Sociologie du tourisme et des voyagos, Paris, PUF, Coll. Ques sais-je?, $1995_{1}$

(2) Ibid.

(3) NADEAU, Roger, Le tourisme: aspects theoricues et pratiques au Quebec, Montreal, Sodilis. 1992

4) Etude réalisete a titre de utravail pratique dans le cadre d'un programme de maitrise en anthropologie a I'Universitu de Montréal

(5) BAFDIN, L., Les mácanismes ideologiques de la publicite, Paris, Jean-Pierre Delarge Editeur. 1975, p. 21

(6) LERIVERAY, Bernard, Guides Bleus, guidas Varts et lunettes roses, Paris, Les Éditions du Cerf, 1975 .

(7) BARDIN, L., L'analyse de contenu, Paris, PUF, 1977 , p. 35

(B) MINISTERE DU TOURISME DU OUEBEC. Plan marketing 1989-1990. Montréal, Direction gunerale du marketing, 1999.

SAMSON BELAIP, Planifieation strategique de marketing du tourisme québecols 19a9-1992, Montréal, $198 \mathrm{~B}$

(9) Ibid.

(10) Op. cit. 